\title{
Dinâmica de eliminação de oocistos de Eimeria sp. durante a gestação e fase inicial da lactação em cabras nativas criadas extensivamente em região semiárida
}

\author{
Dynamic of "Eimeria" oocyst excretion during pregnancy and early lactation in native \\ goats raised extensively in semiarid region
}

\author{
NUNES, Deisiane Moreira ${ }^{1 *}$; CRUZ, Jurandir Ferreira ${ }^{2}$; TEIXEIRA NETO, Milton \\ Rezende 3
}

\author{
${ }^{1}$ Universidade Estadual do Sudoeste da Bahia, Programa de Pós-Graduação em Zootecnia, Itapetinga, \\ Bahia, Brasil. \\ ${ }^{2}$ Universidade Estadual do Sudoeste da Bahia, Departamento de Fitotecnia e Zootecnia, Vitória da \\ Conquista, Bahia, Brasil. \\ ${ }^{3}$ Faculdade de Tecnologia e Ciências, Vitória da Conquista, Bahia, Brasil. \\ *Endereço para correspondência: deisiane.ms@ @otmail.com
}

\section{RESUMO}

Objetivou-se com este estudo caracterizar a dinâmica de eliminação de oocistos de Eimeria sp. em cabras sem raça definida (SRD), criadas em sistema extensivo na região semiárida, durante a gestação e fase inicial da lactação. Vinte cabras adultas, criadas extensivamente na Caatinga, foram selecionadas e monitoradas durante oito meses. Os valores de Oopg (quantidade de oocistos por grama de fezes) foram acompanhados quinzenalmente do $1^{\circ}$ mês de gestação ao $90^{\circ}$ dia pós-parto. O Oopg variou durante a gestação, sendo as maiores e menores contagens de $3.300 \pm 709$ e $1.726 \pm 215$, respectivamente. Após elevação na primeira quinzena, o Oopg diminuiu gradualmente até o $105^{\circ}$ dia e voltou a subir até o final da gestação. No período pós-parto, seguindo a tendência da fase final da gestação, houve aumento progressivo na eliminação de oocistos até o $90^{\circ}$ dia de lactação. A quantidade média de oocistos liberados durante a fase inicial da lactação foi de $3.006 \pm 102,3$ Oopg comparado ao período gestacional de $2.312 \pm 98,03$ Oopg $(\mathrm{P}<0,05)$. A dinâmica de eliminação de oocistos de Eimeria sp. em cabras gestantes e lactantes, criadas extensivamente em região semiárida, foi caracterizada por três situações distintas: elevação brusca no início da gestação, seguida por redução gradual no período intermediário da gestação e aumento constante da eliminação de oocistos na fase final da gestação até inicial da lactação.

Palavras-chave: cabras gestantes, Oopg, pósparto

\section{SUMMARY}

The study aimed to characterize the excretion dynamic of Eimeria oocyst during pregnancy and early lactation in native goats raised extensively in semiarid region. Twenty pregnancy does were selected and monitored during eight months. The count of oocyst per gram of feces (Oopg) was determined fortnightly from first month of pregnancy until $90^{\circ}$ day postpartum. The Oopg varied during pregnancy and the high stand lowest values were $3,300 \pm 709$ and $1,726 \pm 215$ respectively. After rise in the first fortnight, the Oopg gradually decreased until the $105^{\text {th }}$ day and increased again until the end of pregnancy. In the post partum period, following the trend of late pregnancy, there was a progressive increase in oocysts excretion until $90^{\text {th }}$ day of lactation. The average number of oocysts excreted during early lactation was greater than during pregnancy, whose values were $3,006 \pm 102.3$ vs $2,312 \pm 98.03$ Oopg, respectively $(\mathrm{P}<0.05)$. The excretion dynamics of oocysts of Eimeria sp. during pregnancy and early lactation in native goats raised extensively in semiarid region is characterized as a sharp rise in the first fortnight, followed by gradual reduction until intermediate phase and a continuous rise in the last third of gestation and early lactation.

Keywords: pregnant goats, Oopg, postpartum 
Rev. Bras. Saúde Prod. Anim., Salvador, v.16, n.1, p.190-198 jan./mar., 2015 http://www.rbspa.ufba.br ISSN 15199940

\section{INTRODUÇÃO}

A coccidiose é uma doença causada por protozoários parasitas do gênero Eimeria, que afetam uma variedade de animais, incluindo pequenos ruminantes (CAVALCANTE et al., 2012). Embora seja uma doença essencialmente de animais jovens, em determinadas circunstâncias, a doença pode atingir animais adultos, principalmente quando ocorre alta densidade populacional, doses maciças de oocistos no ambiente, estresse, doenças concomitantes e ausência ou queda de imunidade (LIMA, 2004).

O fenômeno conhecido como spring rise, que se refere ao aumento da carga parasitária e consequente eliminação de ovos de helmintos nas fezes durante o período do periparto (RAHMAN \& COLLINS, 1992), tem sido amplamente relatado em ovelhas (GONZALES et al., 1991) e vacas (FABER et al., 2002). Entretanto, a ocorrência desse fenômeno na eliminação de oocistos de Eimeria sp. na espécie caprina não está devidamente esclarecido.

Alguns estudos têm relatado que a eliminação de oocistos pelas cabras gestantes e/ou lactantes pode estar associada à condição fisiológica dos animais (HASSUM \& MENESES, 2005). No entanto, os mecanismos pelos quais o fenômeno ocorre ainda são desconhecidos (FABER et al., 2002; COSTA et al., 2011). A eliminação de oocistos pelas fêmeas no período do periparto é um fator que contribui para o aumento na contaminação ambiental (FABER et al., 2002) e, consequentemente, constitui uma fonte de infecção para os animais jovens (HASSUM et al., 2002; CAVALCANTE et al., 2012).

A compreensão da dinâmica dos valores de Oopg em cabras, durante a gestação e lactação, possui a importância de auxiliar na tomada de decisões quanto ao controle estratégico da coccidiose. Diante disso, objetivou-se com este trabalho caracterizar a dinâmica de eliminação dos oocistos de Eimeria sp. em cabras naturalmente infectadas e criadas extensivamente em região semiárida, durante a gestação e fase inicial da lactação.

\section{MATERIAL E MÉTODOS}

O experimento foi realizado em uma propriedade rural do município de Brumado - BA (13³9'13"S, $41^{\circ} 50$ '37'O), a $422 \mathrm{~m}$ de altitude, localizado na região Sudoeste da Bahia, situado na Depressão Sertaneja Meridional do bioma caatinga (VELLOSO et al., 2002).

O clima da região é classificado como semiárido - Bsh, segundo a classificação Köeppen-Geiger (PEEL et al., 2007). O ensaio foi conduzido nos meses de outubro/2012 a maio/2013 (Figura 1), período no qual a temperatura média foi de $28^{\circ} \mathrm{C}$ e precipitação pluviométrica de 289mm (INMET, 2014).

O rebanho era composto por oito reprodutores da raça Boer e 250 cabras sem raça definida (SRD). Foi realizada a monta natural, em estação reprodutiva de 40 dias. Decorridos 30 dias do acasalamento, as fêmeas foram submetidas ao exame de ultrassonografia, via transretal, utilizando aparelho modelo 100 Falco, Pie Medical.

Após a confirmação da gestação, foram selecionadas 20 cabras multíparas, com escore de condição corporal (E.C.C.) médio de 3,0 (escala de 1,0 - 5,0), (MORAND-FEHR \& HERVIEU, 1999). No mês anterior à estação reprodutiva, as cabras receberam tratamento antihelmíntico à base de monepantel (Zolvix®, Novartis, Brasil), em dose 
Rev. Bras. Saúde Prod. Anim., Salvador, v.16, n.1, p.190-198 jan./mar., 2015 http://www.rbspa.ufba.br ISSN 15199940

única de $2,5 \mathrm{mg} / \mathrm{kg}$ peso vivo. Durante o período de realização do estudo, não foi administrado nenhum fármaco anticoccidiano.

As cabras foram mantidas em pastagem nativa da Caatinga, suplementadas no período mais seco do ano com palma forrageira (Opuntia ficcus). Sal mineral (Caprinofós®, Tortuga, Brasil) e água foram fornecidos ad libitum durante todo o período do estudo.

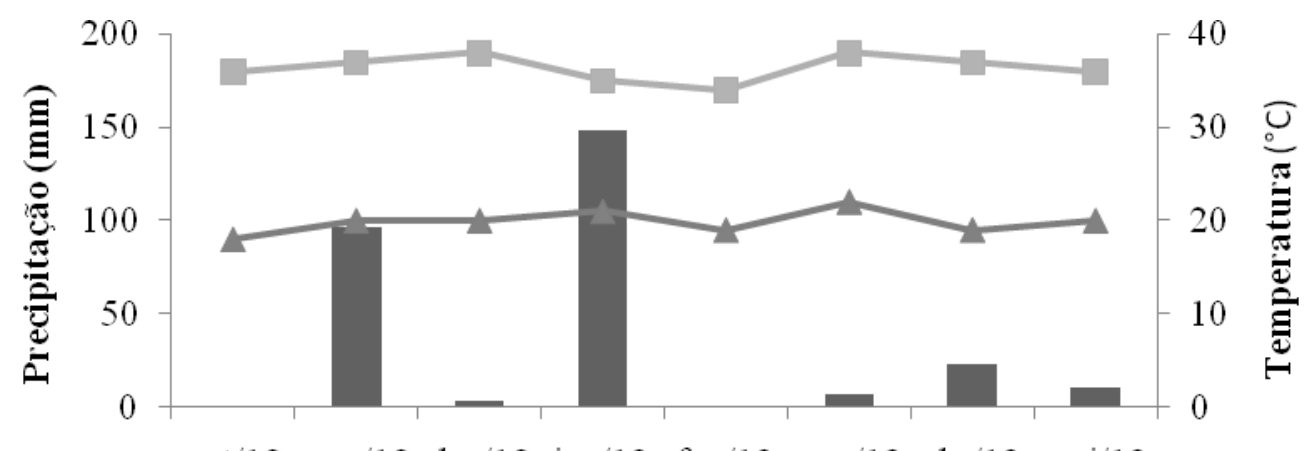

out/12 nov/12 dez/12 jan/13 fev/13 mar/13 abr/13 mai/13

Figura 1. Temperaturas mínima e máxima $(-\boldsymbol{\Lambda}$; - ) e precipitação pluviométrica ( ) no período de outubro/2012 a maio/2013 (INMET, 2014)

As coletas das amostras de fezes foram efetuadas quinzenalmente, do início da estação de acasalamento até o final do estudo, para assegurar o acompanhamento a partir do $1^{\circ}$ mês de gestação até o $3^{\circ}$ mês pós-parto. Cada cabra foi monitorada durante oito meses.

As amostras foram coletadas diretamente da ampola retal, armazenadas em sacos plásticos individualmente identificados, acondicionados em recipiente isotérmico contendo gelo reciclável e enviadas imediatamente ao laboratório. As análises foram realizadas no Laboratório de Biologia do Instituto Federal de Educação, Ciência e Tecnologia Baiano - Campus Itapetinga, até 48 horas após a coleta.

As amostras fecais foram processadas de acordo com a técnica McMaster de flutuação, desenvolvida por Gordon \& Whitlock (1939), e modificada por Ueno \& Gonçalves (1998). Dois gramas de fezes de cada amostra foram maceradas e diluídas em $58 \mathrm{~mL}$ de solução saturada de cloreto de sódio e a suspensão resultante foi filtrada em peneira de malha fina; uma pequena alíquota foi coletada e utilizada para preencher as retículas da câmara de McMaster; a leitura foi procedida em microscópio óptico (40X) e a quantidade de oocistos por grama de fezes (Oopg) foi determinada pelo número de oocistos de Eimeria sp encontrados x 100.

$\mathrm{Na}$ análise dos resultados, os dados referentes ao Oopg foram previamente transformados em logaritmos. Os dados (médias \pm EP) de Oopg dos diferentes períodos de gestação (inicial, intermediário e final) e lactação (período inicial) foram comparados pelo teste de Student-Newman-Keuls do PROC GLM, SAS versão 9.1 (STATISTICAL ANALYSIS SYSTEM, 2003). As médias de Oopg da gestação e lactação foram comparadas pelo teste $\mathrm{t}$ de Student (EXCEL, 2007). As diferenças foram consideradas significativas com $\mathrm{P}<0,05$. 
Rev. Bras. Saúde Prod. Anim., Salvador, v.16, n.1, p.190-198 jan./mar., 2015 http://www.rbspa.ufba.br

\section{RESULTADOS E DISCUSSÃO}

Todas as cabras eliminaram oocistos de Eimeria sp. nas fezes durante todas as fases da gestação, sendo que $45 \%$ das cabras apresentaram amostras contendo mais de $4.10^{3}$ Oopg na fase inicial da gestação.

O sistema de criação extensivo constituiu em um fator de redução do grau de exposição dos animais aos parasitas. No entanto, o habitual recolhimento do rebanho ao final do dia para pernoite nas instalações, certamente, elevou a contaminação ambiental (LIMA, 2004) e favoreceu a reinfecção devido à ingestão de oocistos esporulados (CHARTIER \& PARAUD, 2012) ao longo da gestação.

A contagem de oocistos de Eimeria sp. eliminados pelas fezes variou durante a gestação $(\mathrm{P}<0,05)$, sendo que os valores médios, mais elevados (3.300 \pm 709 Oopg) e mais baixos (1.726 \pm 215 Oopg), ocorreram aos 15 dias (período inicial) e 105 dias (período intermediário) de gestação, respectivamente (Tabela 1).

Tabela 1. Quantidade de oocistos eliminados por grama de fezes (Oopg) ao longo da gestação de cabras SRD, naturalmente infectadas e criadas em região semiárida

\begin{tabular}{lccc}
\hline \multirow{2}{*}{ Dias de gestação } & \multicolumn{3}{c}{ Oopg } \\
\cline { 2 - 4 } & Média $\pm \mathrm{EP}$ & Mínimo & Máximo \\
\hline 0 & $2.343 \pm 452^{\mathrm{AB}}$ & 600 & 6800 \\
15 & $3.300 \pm 709^{\mathrm{A}}$ & 600 & 14700 \\
30 & $2.383 \pm 281^{\mathrm{AB}}$ & 600 & 5000 \\
45 & $2.716 \pm 236^{\mathrm{AB}}$ & 700 & 4400 \\
60 & $2.160 \pm 226^{\mathrm{AB}}$ & 400 & 3500 \\
75 & $2.058 \pm 247^{\mathrm{AB}}$ & 300 & 3800 \\
90 & $1.850 \pm 274^{\mathrm{AB}}$ & 200 & 4200 \\
105 & $1.726 \pm 215^{\mathrm{B}}$ & 800 & 3600 \\
120 & $2.030 \pm 165^{\mathrm{AB}}$ & 400 & 3100 \\
135 & $2.450 \pm 189^{\mathrm{AB}}$ & 500 & 3700 \\
150 & $2.460 \pm 207^{\mathrm{AB}}$ & 400 & 3800 \\
\hline
\end{tabular}

$\overline{\mathrm{A}, \mathrm{B}}$ Valores com letras diferentes diferem pelo teste Student-Newman-Keuls $(\mathrm{P}<0,05)$.

O efeito das infecções por Eimeria sp. é dependente, principalmente, da combinação do nível de contaminação ambiental (GREGORY et al., 1983) com os aspectos imunológicos do hospedeiro (RUIZ et al., 2014) e, durante a gestação, ocorre a diminuição da resistência das matrizes a helmintos, vírus, bactérias e protozoários (LLOYD, 1983).

A estação de acasalamento pode ser uma causa de estresse para fêmeas e machos e, consequentemente, um fator que interfere negativamente sobre $o$ estado fisiológico do animal (FAYER, 1980). A ocorrência do pico de eliminação de oocistos imediatamente após a concepção ( $15^{\circ}$ dia de gestação) pode ter sido reflexo da soma do estresse resultante da estação de acasalamento e exposição ao parasita, em adição à natural imunossupressão decorrente da elevação do nível 
Rev. Bras. Saúde Prod. Anim., Salvador, v.16, n.1, p.190-198 jan./mar., 2015 http://www.rbspa.ufba.br ISSN 15199940

plasmático da progesterona (LLOYD, 1983).

Por outro lado, do $15^{\circ}$ ao $105^{\circ}$ dia de gestação, houve decréscimo gradual dos valores de Oopg (Figura 2). Esse fato, que pode ter sido decorrente do momento fisiológico de baixo estresse, contribuiu para o alcance do equilíbrio hospedeiro/parasito (PINTO et al., 2008), reduzindo a contaminação ambiental e a reinfecção dos animais (LIMA, 2004).
No terço final da gestação $\left(105^{\circ}\right.$ a $150^{\circ}$ dia) ocorreu elevação gradual do Oopg, cujos níveis de eliminação retornaram aos verificados no período inicial $\left(30^{\circ}\right.$ $45^{\circ}$ dia) da gestação (Figura 2). O aumento na eliminação dos oocistos com aproximação do parto foi observado em estudos prévios em vacas (FABER et al., 2002) e coelhas (PAPESCHI et al., 2013).

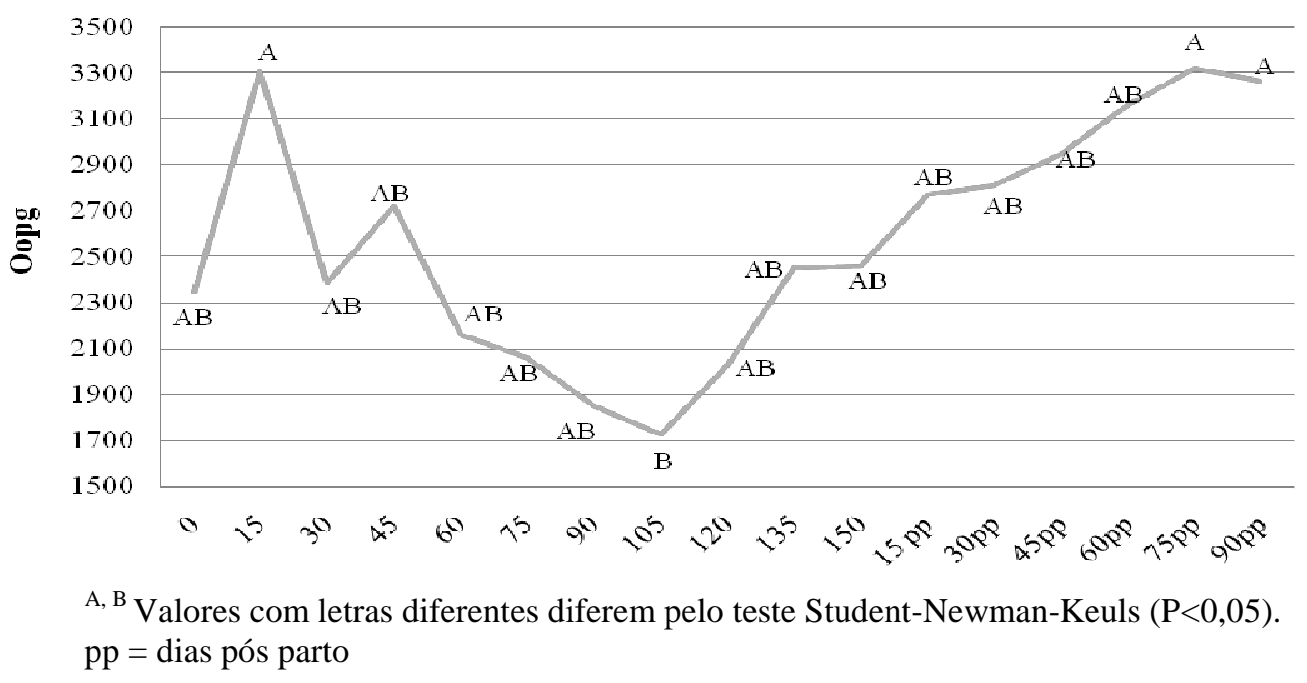

Figura 2. Quantidade de oocistos de Eimeria sp por grama de fezes (Oopg) durante a gestação e início da lactação em cabras sem raça definida (SRD) criadas em sistema extensivo na Caatinga

Em relação as oscilações, a intensidade de infecção durante a gestação e a lactação foi moderada e variou entre $1.10^{3}-1.10^{4}$ Oopg. Por outro lado, a quantidade média de oocistos liberados durante a fase inicial da lactação foi de $3.006 \pm 102,3$ Oopg, superior quando comparada a contagem de todo $\mathrm{o}$ período gestacional, que foi de $2.312 \pm$ 98,03 Oopg (Figura 3; $\mathrm{P}<0,05$ ).

A diminuição da imunidade no período periparto parece estar mais associada com a lactação do que com a prenhez, ainda que o fenômeno seja iniciado no final da gestação (BARGER, 1993). O aumento nos requerimentos nutricionais durante a gestação e lactação é outro fator que pode ter um papel relevante na queda da resistência contra as infecções parasitárias (PAPESCHI, et al., 2013).

Durante as últimas semanas da gestação e as primeiras da lactação, as fêmeas necessitam dobrar o seu consumo de proteína e energia (LEITE, 2002) e há evidências do envolvimento desses parâmetros na quebra da imunidade no período periparto de cabras (ETTER et al., 1999). No presente estudo, as cabras foram suplementadas com palma forrageira (Opuntia ficcus) no período 
Rev. Bras. Saúde Prod. Anim., Salvador, v.16, n.1, p.190-198 jan./mar., 2015 http://www.rbspa.ufba.br ISSN 15199940

mais seco do ano. No entanto, este alimento apresenta limitações quanto ao valor proteico e de fibra, não atendendo às necessidades nutricionais das fêmeas em reprodução (ALMEIDA, 2012). No decorrer da gestação, ocorreram três momentos distintos na eliminação de oocistos. Inicialmente, elevação do Oopg na primeira quinzena; redução na fase intermediária e elevação na fase final (Figura 4). No período pós-parto, seguindo a tendência da fase final da gestação, houve aumento progressivo na eliminação de oocistos até o $90^{\circ}$ dia de lactação, quando alcançou valor similar ao observado na primeira quinzena da gestação (Tabela 2).

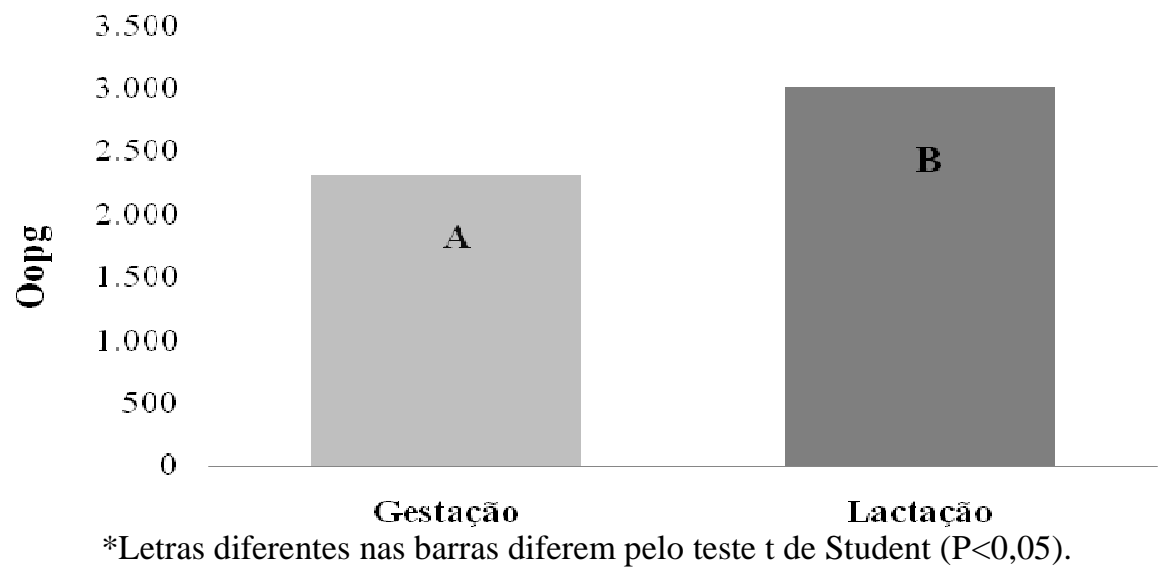

Figura 3. Quantidade média de oocistos de Eimeria sp. eliminados durante a gestação e fase inicial da lactação em cabras sem raça definida (SRD) criadas extensivamente em região semiárida

Tabela 2. Quantidade de oocistos por grama de fezes (Oopg) na fase inicial da lactação em cabras sem raça definida (SRD) criadas extensivamente em região semiárida

\begin{tabular}{cccc}
\hline \multirow{2}{*}{ Dias pós-parto } & \multicolumn{3}{c}{ Oopg } \\
\cline { 2 - 4 } & Média $\pm \mathrm{EP}$ & Mínimo & Máximo \\
\hline 15 & $2.770 \pm 214^{\mathrm{AB}}$ & 500 & 3900 \\
30 & $2.810 \pm 182^{\mathrm{AB}}$ & 1100 & 4100 \\
45 & $2.944 \pm 218^{\mathrm{AB}}$ & 1600 & 4400 \\
60 & $3.156 \pm 251^{\mathrm{AB}}$ & 700 & 4500 \\
75 & $3.319 \pm 313^{\mathrm{A}}$ & 500 & 4900 \\
90 & $3.263 \pm 456^{\mathrm{A}}$ & 1100 & 5300 \\
\hline
\end{tabular}

${ }^{\mathrm{A}, \mathrm{B}}$ Valores com letras diferentes diferem pelo teste Student-Newman-Keuls $(\mathrm{P}<0,05)$.

A maior eliminação de oocistos durante a lactação foi observada em estudos prévios, os quais ressaltaram a estreita relação entre a intensidade de infecção por Eimeria sp e o estado fisiológico das cabras leiteiras (MENESES \& LOPES, 1997; CHARTIER et al., 1998).
Quaisquer condições que rompam o equilíbrio hospedeiro/parasito podem promover incremento na eliminação de oocistos (PINTO et al., 2008).

A eliminação de maiores quantidades de oocistos durante a lactação eleva a contaminação ambiental e, 
Rev. Bras. Saúde Prod. Anim., Salvador, v.16, n.1, p.190-198 jan./mar., 2015 http://www.rbspa.ufba.br ISSN 15199940

consequentemente, aumenta o grau de exposição dos animais neonatos (JOLLEY \& BARDSLEY, 2006). Por outro lado, fatores ambientais como a prática de desmame podem contribuir para imunosupressão dos cabritos e assim potencializar infecções latentes (CHARTIER \& PARAUD, 2012; LIMA, 2014). Dessa forma, é atribuído às mães o importante papel na contaminação ambiental e transmissão dos oocistos, confirmando que estas podem se tornar a principal fonte de infecção para os animais jovens, os quais constituem a categoria mais susceptível a coccidiose (VIEIRA et al., 1999).

A dinâmica de eliminação de oocistos de Eimeria sp. em cabras gestantes e lactantes, criadas extensivamente em região semiárida, foi caracterizada por três situações distintas: elevação brusca no início da gestação, seguida por redução gradual no período intermediário e aumento constante da eliminação de oocistos da fase final da gestação até inicial da lactação.

A elevada eliminação de oocistos na fase final da gestação e inicial da lactação torna imperativo o monitoramento $\mathrm{e}$ controle da coccidiose nas matrizes, no período periparto.

\section{REFERÊNCIAS}

ALMEIDA, R.F. Palma forrageira no alimentação de ovinos e caprinos no semiárido brasileiro.Revista Verde de Agroecologia e Desenvolvimento Sustentável, v.7, n.4, p.08-14, 2012.

BARGER, A.I. Influence of sex and reproductive status on susceptibility of ruminants to the nematode parasitism.

International Journal for Parasitology, v.23, n.4, p.463-469, 1993.
CAVALCANTE, A.C.R.; TEIXEIRA, M.; MONTEIRO, J.P.; LOPES, C.W.G. Eimeria species in dairy goats in Brazil. Veterinary Parasitology, v.183, p.356358, 2012.

CHARTIER, C.; HOSTE, H.;

BOUQUET, W.; MALPAUX, B; PORS, I.; KOCH, C. Periparturient rise in fecal egg counts associated with prolactin concentration increase in French Alpine dairy goats.

Parasitology Research, v.84, p.806810, 1998.

CHARTIER, C.; PARAUD, C. Coccidiosis due to Eimeria in sheep and goats, a review. Small Ruminants

Research, v.103, n.1, p.84-92, 2012.

COSTA, V.M.M.; SIMÕES, S.V.D.; RIET-CORREA, F. Controle das parasitoses gastrintestinais em ovinos e caprinos na região semiárida do Nordeste do Brasil. Pesquisa Veterinária Brasileira, v.31, n.1, p.6571, 2011.

ETTER, E.; CHARTIER, C.; HOSTE, H.; PORS, I.; BOUQUET, W.; LEFRILEUX, Y.; BORGIDA, L. P. The influence of nutrition on the periparturient rise in fecal egg counts in dairy goats: results from a two-year study. Revue de Medecine

Veterinaire, v.150, n.12, p.975-980, 1999.

FABER, J.E.; KOLLMANN, D.; HESE, A. BAUER, C. FAILING, K.; BÜRGER, H.J.; ZAHNER, H. Eimeria infections in cows in the periparturient phase and their calves: oocyst excretion and levels of specific serum and colostrums antibodies. Veterinary Parasitology, v.104, p.1-17, 2002. 
Rev. Bras. Saúde Prod. Anim., Salvador, v.16, n.1, p.190-198 jan./mar., 2015 http://www.rbspa.ufba.br ISSN 15199940

FAYER, R. Epidemiology of protozoan infections: the coccidia. Veterinary Parasitology, v.6, n.1, p.75-103, 1980.

GONZALES, J.M.; SANCHEZ, A.A.; VASQUES, V.P. Presence and dynamics of oocysts of some species of Eimeria in ewes and lambs during the perinatal period in Huixquilican, México. Protozool Abstr, v.15, p. 225228, 1991.

GORDON, H. M.; WHITLOCK, H. V. A. New technique for counting nematodes eggs in sheep faeces.

Journal of the Council for Scientifc and Industrial Research, v.12, p.5052, 1939.

GREGORY, M.W.; CATCHPOLE, J.; JOYNER, L.P.; PARKER, B.N.J.

Observations on the epidemiology of coccidial infections in sheep under varying conditions of intensive husbandry including chemoprophylaxis with monensin. Parasitology, v.87, p.421427, 1983.

HASSUM, I.C.; MENESES, R.C.A.A. Infecção natural por espécies do gênero Eimeria em pequenos ruminantes criados em dois municípios do estado do Rio de Janeiro. Revista Brasileira de Parasitologia Veterinária, v.14, n.3, p.95-100, 2005.

HASSUM, I.C.; PAIVA, R.V.; MENEZES, R.C.A.A. Frequência, dinâmica e morfologia dos oocistos de Eimeriabakuensis (Apicomplexa: Eimeriidae) em ovinos de diferentes categorias de produção de uma criação no município de Petrópolis/RJ. Revista Brasileira de Parasitologia Veterinária, v.11, n.1, p.19-25, 2002.
INSTITUTO NACIONAL DE METEOROLOGIA - INMET. Estações e Dados. Disponível em <www.inmet.gov.br>. Acesso em: 18 maio 2014.

JOLLEY, W.R.; BARDSLEY,K.D. Ruminant Coccidiosis. Veterinary Clinics Food Animal Pratice, v.22, p.613-621, 2006.

LEITE, E.R. Manejo alimentar de caprinos e ovinos em pastejo no Nordeste do Brasil. Ciência Animal, v.12, n.2, p.119-128, 2002.

LIMA, J.D. Coccidiose dos Ruminantes Domésticos. Revista Brasileira de Parasitologia Veterinária, v.13, p.9-13, 2004. Supl. 1.

LLOYD, S.S. Immunossupression during pregnance and lactation. Irish

Veterinary Journal, v.37, p.64-70, 1983.

MENESES, R.C.A.A., LOPES, C.W.G.

Eimeria alijevi (Apicomplexa:

Eimeriidae) em caprinos leiteiros na microrregião serrana fluminense, RJ. Revista Brasileira de Parasitologia Veterinária, v.6, n.1, p.66-73, 1997.

MORAND-FEHR, P.; HERVIEU, J. Apprécier l'éat corporel des chèvres: Intérêt et méthod. Reussir La Chevre, v.231, p.22-34, 1999.

PAPESCHI, C.; FICHI, G.; PERRUCCI, S. Oocysts excretion pattern of three intestinal Eimeria species in female rabbits. World Rabbit Science, v.21, p.77-83, 2013.

PEEL, M.C.; FINLAYSON, B.L.; MCMAHON, T.C. Updated world map of the Koppen-Geiger climate classification. Hydrology and Earth System Sciences, v.11, p.1633-1644, 2007. 
Rev. Bras. Saúde Prod. Anim., Salvador, v.16, n.1, p.190-198 jan./mar., 2015 http://www.rbspa.ufba.br

PINTO, J.M.; OLIVEIRA, M.A.L.; ÁLVARES, C.T.; COSTA-DIAS, R.; SANTOS, M.H. Relação entre o periparto e a eliminação de ovos de nematoides gastrintestinais em cabras anglo nubiana naturalmente infectadas em sistema semi-extensivo de produção. Revista Brasileira de Parasitologia Veterinária, v.17, p.138-143, 2008. Supl. 1.

RAHMAN, W.A.; COLLINS, G.H. An association of faecal egg counts and prolactin concentrations in sera of periparturient Angora goats. Veterinary Parasitology, v.43, n.1, p.85-91, 1992.

RUIZ, A.; MUÑOZ, M.C.; MOLINA, J.M.; HERMOSILLA, C.; ANDRADA, M.; LARA, P.; BORDÓN, E.; PÉREZ, D.; LÓPEZ, A.M.; MATOS, L.; GUEDES, A.C.; FALCON, S.; FALCÓN, Y.; MARTIN, S.; TAUBERT, A. Immunization with Eimeria ninakohlyakimovae -live attenuated oocysts protect goat kids from clinical coccidiosis. Veterinary Parasitology, v.199, p.8-17, 2014.

STATISTICAL ANALYSIS SYSTEM. SAS user's guide. Version 9.1 ed. Cary: SAS Institute, USA: SAS Institute Inc, 2003.

UENO, H.; GONÇALVES, P.C. Manual para o diagnóstico das helmintoses de ruminantes. 4.ed. Tokyo: Japan International Cooperation Agency, 1998. 143p.

VIEIRA, L.S.; CAVALCANTE, A.C.R.; XIMENES, L.J. F. Evolution of infection with Eimeria species in hair sheep reared in Sobral, Ceara State, Brazil. Revue de Médecine Vétérinaire, v.150, n.6, p.547-550, 1999.
VELLOSO, A.L.; SAMPAIO, E.V.S.; PAREYN, F S.G.C. Ecorregiões propostas para o bioma Caatinga. Recife: Associação Plantas do Nordeste, 2002. 76 p.

Data de recebimento: 13/10/2014

Data de aprovação: 06/03/2015 